\title{
Sidelobe Reduction in the Pulse-Compression Radar Using Synthesis of NLFM Laws
}

\author{
Iulian-Constantin Vizitiu \\ Department of Communications and Military Electronic Systems, Military Technical Academy, George Coşbuc Avenue 39-49, \\ 050141 Bucharest, Romania \\ Correspondence should be addressed to Iulian-Constantin Vizitiu; iulian.vizitiu@gmail.com
}

Received 24 October 2012; Revised 28 January 2013; Accepted 18 February 2013

Academic Editor: Stefano Selleri

Copyright (C) 2013 Iulian-Constantin Vizitiu. This is an open access article distributed under the Creative Commons Attribution License, which permits unrestricted use, distribution, and reproduction in any medium, provided the original work is properly cited.

It is well known that in the pulse-compression radar theory, the sidelobe reduction using synthesis of some proper nonlinear FM (NLFM) laws represents a major research direction. In order to assure the sidelobe suppression, the main objective of this paper is to present an adequate synthesis algorithm of NLFM signals based on stationary phase principle. The achieved experimental results confirm a significant sidelobe reduction (i.e., more than $-40 \mathrm{~dB}$ ) without necessity to apply some weighting techniques. In addition, the analysis of the synthesized NLFM laws by ambiguity function tool is also discussed.

\section{Introduction}

According to the literature $[1,2]$, it is well known that the signal (pulse-) compression techniques are used inside of all modern radar systems to increase the range resolution without having to increase the peak transmit power. Whereby the range resolution is inverse proportional with the frequency band of the scanning signals, in the last period of time, in radar system theory, a lot of suitable wideband signals (e.g., short radio pulse, signals with discrete frequency modulation, signals with LFM or NLFM, unsinusoidal signals, etc.) were designed and analyzed as performance level (e.g., using the well-known ambiguity function tool).

Generally, one of the most important requests imposed to the wideband radar signals is to assure the lowest level of the sidelobes assigned to the response of the compression (matched) filter. According to [3], the presence in the response of significant sidelobes may cause interference with the other near echo signals and having unwanted effects in the detection process and ambiguities in the estimating of the range targets. Therefore, an important research direction in the literature refers to the design of improved methods to synthetize radio pulses with rectangular envelope, but with suitable modified FM laws (i.e., NLFM signals) so that the matched filter response becomes one closer by the expected values [4].

The NLFM signals are other continuous phase modulation waveforms with applicability inside of pulsecompression radar systems. They have been claimed to provide a high-range resolution, an improved signal to noise ratio (SNR), low cost, and good interference mitigation. In addition, they have a spectrum weighting function inherently in their modulation function, which offers the advantage that a pure matched filter gives low sidelobes (thus, the loss in SNR associated with weighting or with the usual mismatching techniques is eliminated). According to [5], the NLFM waveforms also assure the better detection rate characteristics and is more accurate in range determination than other consecrated processing methods (e.g., dual apodization (DA), spatially variant apodization (SVA), leakage energy minimization (LEM), etc.).

In radar theory, there are many interesting scientific research works which have been done to investigate the scanning waveforms and attempt to design optimal (i.e., as level of the sidelobe suppression) NLFM signals [6-9]. However, all these processing methods can be generally divided into two major research directions, namely: (a) design and synthesis of pseudo-NLFM waveforms which are in fact signals with LFM 
predistortioned on short intervals into temporal domain or corrected into spectral domain [10-12]; (b) design and synthesis of purely (i.e., as predefined shape of the energy/power spectral density (E/PSD) function) NLFM waveforms using, for example, proper iterative methods $[3,11]$, stationary phase principle [13-16], or explicit functions cluster method [17, 18], and so forth. In addition, the most part of the present processing methods used to assure the sidelobe reduction belonging to standard computational techniques [6-18], but some interesting aspects connected with artificial intelligence paradigms are also discussed in the literature [19-21].

Referring now to the synthesis of NLFM laws using the stationary phase concept, in the radar literature, several scientific papers containing some interesting results are illustrated [1-4, 13-15]. However, as a common design characteristic, all these synthesis methods have as starting point the choice of a desired spectrum shape (generally, only ordinary window functions), and next they gradually perform the specific calculus of NLFM waveforms, but no complete details about their processing stages, the influence of some external factors (e.g., Doppler frequency shift), or clear ideas about the effective way to set some parameters involved in this design process are indicated.

Beside the main goal to remove the previously mentioned drawbacks, the synthesis algorithm of NLFM laws described in this paper offers some significant advantages related to other approaches, such as its general behavior (i.e., any type of PSD function can be used as input), assures a concrete way to adjust (recalculate) the shape of the synthesized spectrum related to the desired one, gives a solution to find the suitable values assigned to some designing parameters (e.g., to preserve the target detection/ranging quality as in case of the similar standard LFM signal), and allows the possibility to effectively analyse the influence of some noisy factors (e.g., a concrete way to study the influence of Doppler frequency shift or time-delay on the shape assigned to the normalized complex envelope of the synthesized signal autocorrelation function).

This paper is aimed to present a proper sidelobe reduction technique based on synthesis of NLFM laws using the stationary phase concept. Consequently, in the first part of the paper, a theoretical overview of the stationary phase principle is indicated. Next, the concrete anatomy of the proposed NLFM synthesis technique is described. In the last part of the paper, some interesting experimental results proving the broached theoretical aspects from beginning are presented. Finally, the most important conclusions are also discussed.

\section{An Overview of the Stationary Phase Principle}

According to [3], beside other well-known processing methods (e.g., explicit functions cluster method), the stationary phase principle-based technique is one of the most important synthesis tool of the complex modulated radar signals (e.g., NLFM signals) with a predefined shape of PSD function. In addition, using analytic or numerical computations, this technique allows obtaining proper frequency modulation

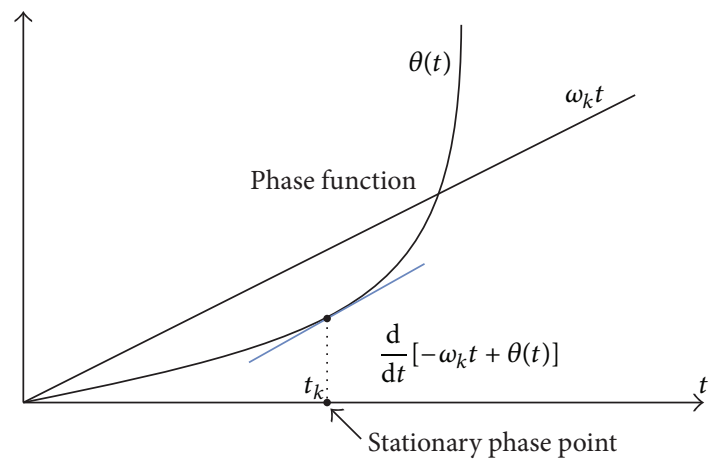

FIGURE 1: The criterion of stationary phase (the stationary point is achieved for $t=t_{k}$ or $\omega_{k}$, when the tangent at function $\theta(t)$ is parallel to straight line of $\omega t$ ).

laws of signals which can assure the desired response of the compression-weighting filter (i.e., in sense of the sidelobe reduction).

Having as starting point the standard representations of a certain signal (with a relative narrow bandwidth) through

its complex envelope $S(t)=A(t) \cdot \exp [j \theta(t)]$ or spectral density function $S(j \omega)=|S(j \omega)| \cdot \exp [j \Phi(\omega)]$, the relations between these can be expressed using well-known pair of Fourier transforms; namely,

$$
\begin{aligned}
S(j \omega) & =\int_{-\infty}^{+\infty} A(t) \cdot \exp \{j[-\omega t+\theta(t)]\} \mathrm{d} t \\
& \stackrel{F T s}{\Longleftrightarrow} S(t)=\frac{1}{2 \pi} \int_{-\infty}^{+\infty}|S(j \omega)| \cdot \exp \{j[\omega t+\Phi(\omega)]\} \mathrm{d} \omega .
\end{aligned}
$$

Assimilating the integral defined from functions by real variable with the notion of area, the periodical functions from (1) show that the significant value of these two integrals is given by the temporal zones (from dynamics of signal wave form) in which the argument of periodical functions is slowly changed with the speed of $(\mathrm{d} / \mathrm{d} t)[-\omega t+\theta(t)]=0$ and $(\mathrm{d} / \mathrm{d} \omega)[\omega t+\Phi(t)]=0$. The points in which the change speed is canceled are named stationary phase points (Figure 1), and these are solutions of the following equations:

$$
\begin{gathered}
\frac{\mathrm{d}}{\mathrm{d} t}[-\omega t+\theta(t)]=0 \Longleftrightarrow \theta^{\prime}(t)=\omega=2 \pi f, \\
\frac{\mathrm{d}}{\mathrm{d} \omega}[\omega t+\Phi(\omega)]=0 \\
\Longleftrightarrow \Phi^{\prime}(\omega)=-t \Longleftrightarrow T_{g}(\omega)=-\Phi^{\prime}(\omega)=t,
\end{gathered}
$$

where $T_{g}(\omega)$ is the time of group delay of the signal.

Generally, the stationary phase points (i.e., temporal or into frequency domain) can be used to approximate the PSD function of a signal, when its complex envelope $S(t)$ is known or, respectively, the power temporal density (PTD) function, 
when the signal spectral density function $S(j \omega)$ is also known, based on following approximate computation equations:

$$
\begin{aligned}
& \left|S\left(j \omega_{t}\right)\right|^{2} \cong 2 \pi \cdot \frac{A^{2}(t)}{\left|\theta^{\prime \prime}(t)\right|^{2}}, \\
& A^{2}\left(t_{\omega}\right) \cong \frac{1}{2 \pi} \cdot \frac{|\dot{S}(j \omega)|^{2}}{\left|\Phi^{\prime \prime}(\omega)\right|},
\end{aligned}
$$

where the used notations $\omega_{t}$ and $t_{\omega}$ refer to the fact that the frequency and time are connected through the stationary phase conditions given by (2). In addition, into stationary phase points, it can also be written that

$$
\omega(t)=T_{g}^{-1}(t)
$$

which means that the temporal variation of the frequency law $\omega(t)$ and group delay function $T_{g}(\omega)$ are inverse functions [4].

According to [13], for the majority of frequency modulated signals, the quality of the approximations achieved by stationary phase method increases at the same time with the increase of the product between time width $(T)$ and band width $(B)$ assigned to these (radar) signals.

More theoretical details about the stationary phase method and its applications into synthesis of the complex radar signals can be found in $[3,4,13]$.

\section{Synthesis of NLFM Laws Using the Stationary Phase Principle}

The stationary phase technique allows using a predefined shape of the signal PSD function (thus, a desired response of the compression filter and implicitly a low level of the sidelobes assigned to the signal autocorrelation function will be assured; in addition, by this method, some major disadvantages assigned to the standard weighted filtering can be also removed) to achieve the synthesis of the proper signal (e.g., NLFM) waveforms. Mostly, this method applyied supposes the use of some numerical computing algorithms of Fourier transforms, because the accurate analytical algorithms do not allow resolving of the nonlinear equation [13].

According to [4], if $|S(j f)|^{2}$ is the PSD function of the signal, based on stationary phase method, the time of group delay can be obtained as follows:

$$
\begin{gathered}
\int_{0}^{t} A(\tau) \mathrm{d} \tau=\int_{-\infty}^{f}|S(j \xi)|^{2} \mathrm{~d} \xi \\
\text { or } \quad \int_{0}^{t} A(\tau) \mathrm{d} \tau=\int_{f}^{+\infty}|S(j \xi)|^{2} \mathrm{~d} \xi .
\end{gathered}
$$

Because the solutions achieved by these two equations are complementary (i.e., the frequency modulation laws have the same form, but with opposite slopes), in the next calculus one of them can be used.
Generally, into all our future NLFM synthesis approaches, some working hypotheses will be supposed. Namely,

(h1) the signal envelope is one rectangular (i.e., a radio pulse):

$$
A(t)= \begin{cases}A(=\text { constant }), & t \in[0, T] \\ 0, & \text { otherwise }\end{cases}
$$

(h2) the bandwidth assigned to spectral density envelope is one limited:

$$
S(f) \cong 0, \quad f \notin\left[-\frac{\Delta F}{2}, \frac{\Delta F}{2}\right] ;
$$

(h3) the shape of the signal PSD function is one predefined (i.e., known):

$$
|S(j f)|^{2}=\left|S_{p d}(j f)\right|^{2} ;
$$

(h4) the values assigned to the time of group delay at the frequency range ends are known:

$$
T_{g}\left(-\frac{\Delta F}{2}\right)=0, \quad T_{g}\left(\frac{\Delta F}{2}\right)=T .
$$

To assure the synthesis of some proper (i.e., by sidelobe suppression point of view) signal frequency or phase modulation laws, the proposed designing algorithm contains the following important processing stages, namely:

(s1) having as basic target the sidelobe reduction, the computation and analysis of the idealized shape assigned to the normalized envelope of the signal autocorrelation function $\rho_{i d}(\cdot)$ using the following equation:

$$
\rho_{i d}(\tau)=\frac{\int_{-\Delta F / 2}^{+\Delta F / 2}\left|S_{p d}(j f)\right|^{2} \cdot \exp (j 2 \pi f \tau) \mathrm{d} f}{\int_{-\Delta F / 2}^{+\Delta F / 2}\left|S_{p d}(j f)\right|^{2} \mathrm{~d} f} ;
$$

(s2) to assure a similar range resolution as in case of LFM radio pulse, the correction of the frequency deviation $\Delta F$ by the form

$$
\Delta F_{c}=a \cdot \Delta F,
$$

where $a$ is a suitable chosen constant more than one $(a>1)$;

(s3) using one of the equations from (5) and working hypothesis (h4), the calculus of the dependency between signal time of group delay and frequency $t=t(f) \stackrel{\text { notation }}{=} T_{g}(f)$, into stationary phase points;

(s4) using the stationary phase condition $\Phi_{s}^{\prime}(f)=-2 \pi$. $T_{g}(f)$ from (2) and by integrating, respectively, the calculus of the signal phase-frequency dependencynamely,

$$
\Phi_{s}(f)=-2 \pi \cdot \int_{-\Delta F_{c} / 2}^{f} T_{g}(\xi) \mathrm{d} \xi ;
$$


(s5) using analytical or numerical procedures and (4), the calculus of the temporal frequency modulation law $f(t)$, as an inverse function of signal time of group delay;

(s6) finally, the calculus of the signal phase modulation law $\theta(t)$, using the following well-known equationnamely,

$$
\theta(t)=2 \pi \cdot \int_{0}^{t} f(\xi) \mathrm{d} \xi
$$

The frequency modulation law determined before has a certain degree of approximation given by stationary phase method. Consequently, having as starting point a signal with rectangular envelope and phase modulation law given by (13), the absolute of the estimated spectral density function assigned to this signal $S_{e s}(j \omega)$ can be easily calculated using the first equation from (1). Next, if we try to compare this estimated function with the predefined one from $(8) S_{p d}(j \omega)$, then the superposition between these two spectra is achieved only in the points where the stationary phase condition is accomplished. However, the more signal base is, the more such superposition points are, and so forth.

Consequently, in order to assure the desired sidelobe reduction by a proper shape of signal autocorrelation function, the calculus steps from previously described algorithm must be repeated and reanalyzed. Thus, the normalized complex envelope of the signal autocorrelation function will be successively recalculated according to following equation:

$$
\rho(\tau)=\frac{1}{T}\left|\int_{0}^{T-|\tau|} \exp \{j[\theta(t)-\theta(t-\tau)]\} \mathrm{d} t\right| .
$$

Finally, in order to have a complete view of NLFM signal synthesis process based on stationary phase method, it is interesting to analyse the influence of the Doppler frequency shift $F$ and time-delay on the shape assigned to the normalized complex envelope of the signal autocorrelation function. Consequently, a similar equation with (14) can be written as follows:

$$
\rho(\tau, F)=\frac{1}{T}\left|\int_{0}^{T-|\tau|} \exp \{j[\theta(t)-\theta(t-\tau)+2 \pi F t]\} d t\right|
$$

As mentioned earlier, for the most part of the nonlinear dependencies between signal time of group delay and frequency (as a result of the chosen predefined shape of PSD functions), it is not possible to find by analytical ways, the temporal variations of the frequency and phase for a signal with rectangular envelope. However, these functions can be achieved by numerical solution of some nonlinear equations describing the $T_{g}(f)$ dependency. In addition, by suitable numerical methods, the temporal samples of NLFM signal phase are thus obtained. Finally, based on the recent advances in the field of signal hardware processing, these samples can be next used to generate the proper shape of the signals, for example, by help of the direct digital synthesis of the complex envelope samples of these $[13,15]$.

\section{Experimental Results}

The main objectives of this experimental part were to present in a detailed manner the results achieved by applying the previously described synthesis algorithm on some predefined PSD functions and to make a comparative study between them as performance level (e.g., by view of the sidelobe reduction capacity, the influence of the Doppler frequency shift on the shape assigned to the signal autocorrelation function), respectively.

According to [14], in order to obtain a compressed pulse in the time domain with a low level of the sidelobes, one of the most important requests is to have a signal with a spectrum decreasing towards the band edges, and with reduced discontinuities in the frequency domain. Consequently, in radar theory, a lot of promising PSD functions are indicated, such as $\cos ^{n}$ and $\cos ^{2}$ on pedestal spectra, Taylor, and truncated Gaussian and Blackman-Harris windows.

Having as starting point the experimental results reported in [14-16], it seems that very good results have been obtained in case of Taylor and Blackman-Harris weighting windows as sidelobe reduction techniques, and therefore these two types of PSD functions will be next used for synthesis and comparative analysis of the matched NLFM laws by the previously described algorithm.

4.1. Synthesis and Analysis of NLFM Signal Using a PSD Function by Taylor Type. The PSD function can be written as follows:

$$
\begin{array}{r}
\left|S_{T}(j f)\right|^{2}=S_{T} \cdot\left[1+2 \cdot \sum_{k=1}^{n-1} F_{k} \cdot \cos \left(2 \pi k \cdot \frac{f}{\Delta F_{T}}\right)\right], \\
f \in\left[-\frac{\Delta F_{T}}{2},+\frac{\Delta F_{T}}{2}\right],
\end{array}
$$

where the number $n$ of the series terms and the values assigned to the coefficients $F_{k}, k=\overline{0, n-1}$, are rigorously determined by the requested level of the first sidelobe $\alpha_{1}$, by the following equations:

$$
\begin{gathered}
F_{0}=1, \\
F_{k_{\mid k \neq 0}}=\frac{1}{2} \cdot \frac{(-1)^{k+1}}{\prod_{p=1, p \neq k}^{n-1}\left(1-k^{2} / p^{2}\right)} \cdot \prod_{p=1}^{n-1}\left[1-\frac{k^{2} \sigma^{-2}}{A^{2}+(k-1 / 2)^{2}}\right],
\end{gathered}
$$

where $A=(1 / \pi) \cdot \operatorname{arcosh}\left(10^{\left|\alpha_{1}(\mathrm{~dB})\right| / 20}\right)$ and $\sigma=n /$ $\sqrt{A^{2}+(n-1 / 2)^{2}}$.

The frequency deviation $\Delta F_{T}$ will have such value that the range resolution is not worsened comparing with the case of LFM radio pulse with frequency deviation $\Delta F_{\text {LFM }}$; namely, $\Delta F_{T}=1.66 \cdot \Delta F_{\mathrm{LFM}}$ for $n=6$, and $\Delta F_{T}=1.86 \cdot \Delta F_{\mathrm{LFM}}$ for $n=8$ (Figure 2). 


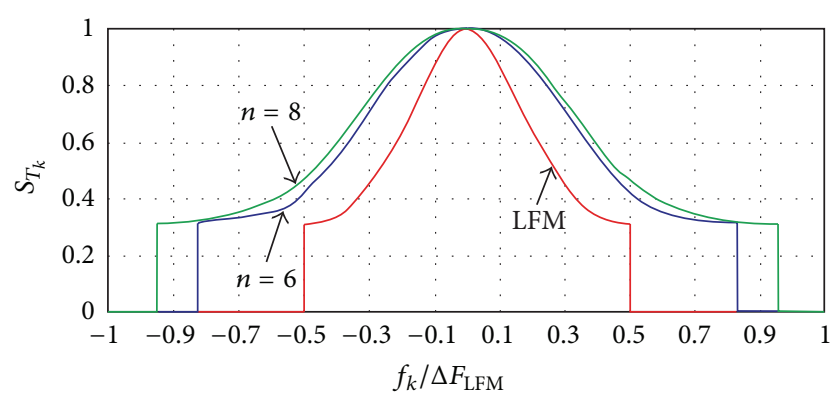

FIGURE 2: Taylor PSD functions.

According to (10), the idealized shape assigned to the normalized envelope of the signal autocorrelation function $\rho_{\mathrm{id}_{T}}(\cdot)$ will be given by the following equation (Figure 3 ):

$$
\begin{aligned}
& \rho_{\mathrm{id}_{T}}(\tau)= \operatorname{sinc}\left(\pi \cdot \tau \cdot \Delta F_{T}\right) \\
&+\sum_{k=1}^{n-1} F_{k}\left\{\operatorname{sinc}\left[\pi\left(\tau \cdot \Delta F_{T}+k\right)\right]\right. \\
&\left.\quad+\operatorname{sinc}\left[\pi\left(\tau \cdot \Delta F_{T}-k\right)\right]\right\} .
\end{aligned}
$$

Next, the time of group delay of the signal can be calculated using the following equation:

$$
\begin{aligned}
T_{g_{T}}(f) & =\int_{-\infty}^{f}\left|S_{T}(j f)\right|^{2} \mathrm{~d} f \\
& =T \cdot\left\{\frac{f}{\Delta F_{T}}+\frac{1}{\pi} \cdot \sum_{k=1}^{n-1}\left[\frac{F_{k}}{k} \cdot \sin \left(2 \pi k \cdot \frac{f}{\Delta F_{T}}\right)\right]+0.5\right\} .
\end{aligned}
$$

It can be easily observed that $T_{g_{T}}\left(-\Delta F_{T} / 2\right)=0$ and $T_{g_{T}}\left(+\Delta F_{T} / 2\right)=T$, respectively (Figure 4$)$.

According to (12), the signal phase-frequency dependency can be written as follows:

$$
\begin{aligned}
\Phi_{s_{T}}(f)=-2 \pi \cdot \int_{-\Delta F_{T} / 2}^{f} T_{g}(\xi) \mathrm{d} \xi \\
=2 \pi \cdot \Delta F_{T} \cdot\left[\frac{1}{2 \pi^{2}}\right. \\
\quad \sum_{k=1}^{n-1} \frac{F_{k}}{k^{2}} \cdot \cos \left(2 \pi k \cdot \frac{f}{\Delta F_{T}}\right) \\
\quad-0.5 \cdot\left(\frac{f}{\Delta F_{T}}\right)^{2}-0.5 \cdot \frac{f}{\Delta F_{T}} \\
\left.\quad-0.125-\frac{1}{2 \pi^{2}} \cdot \sum_{k=1}^{n-1} \frac{F_{k}}{k^{2}} \cdot(-1)^{k}\right] .
\end{aligned}
$$

The frequency modulation law obtained by solving through numerical methods of the equations $T_{g_{T}}(f)=t$ is depicted in Figure 5.

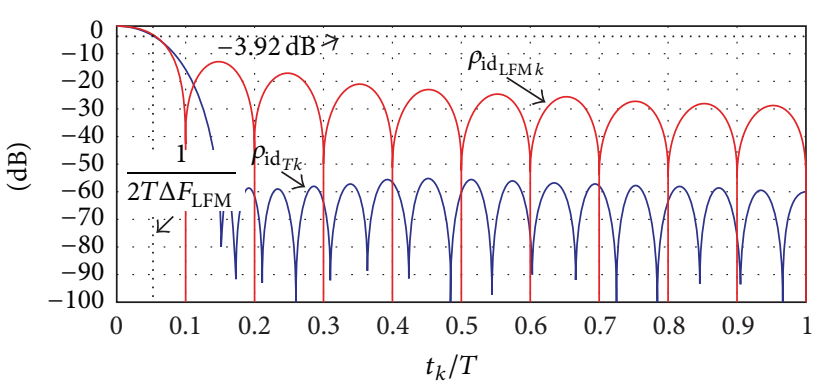

$\Delta F_{\mathrm{LFM}}=1 \mathrm{MHz}, \Delta F_{T}=1.66 \cdot \Delta F_{\mathrm{LFM}}, n=6, \alpha_{1}=-60 \mathrm{~dB}, \mathrm{BT}=100$

(a)

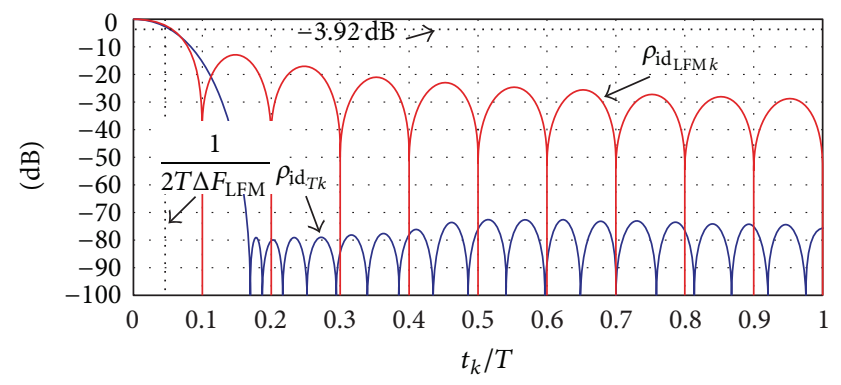

$\Delta F_{\mathrm{LFM}}=1 \mathrm{MHz}, \Delta F_{T}=1.86 \cdot \Delta F_{\mathrm{LFM}}, n=8, \alpha_{1}=-80 \mathrm{~dB}, \mathrm{BT}=100$

(b)

FIGURE 3: The normalized envelope of the signal autocorrelation function using the correction of the frequency deviation.

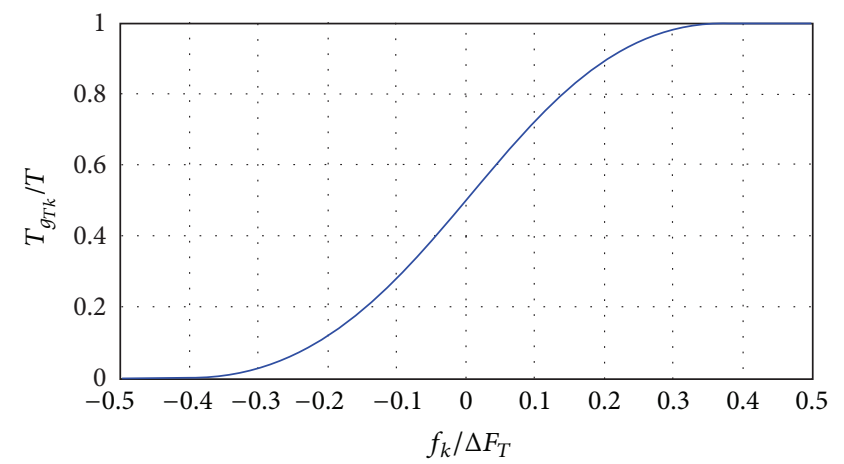

$\Delta F_{\mathrm{LFM}}=1 \mathrm{MHz}, \Delta F_{T}=1.86 \cdot \Delta F_{\mathrm{LFM}}, n=8, \alpha_{1}=-80 \mathrm{~dB}, \mathrm{BT}=100$

FIGURE 4: Dependency between time of group delay and frequency for a signal having a Taylor PSD function.

The phase modulation law obtained by numerical integration of the frequency modulation law can be calculated according to following equations:

$$
\begin{array}{r}
\varphi_{T}(t)=2 \pi \cdot \int_{0}^{t} f_{T}(t) \mathrm{d} t \Longleftrightarrow \varphi_{T_{k}}=2 \pi \cdot \frac{T}{N} \cdot \sum_{i=0}^{k} f_{T_{i}}, \\
k=0,1, \ldots, N,
\end{array}
$$

and is also depicted in Figure 6. 


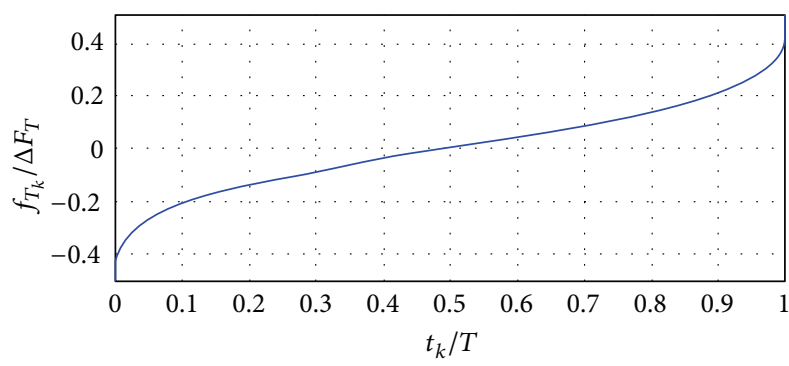

$\Delta F_{\mathrm{LFM}}=1 \mathrm{MHz}, \Delta F_{T}=1.86 \cdot \Delta F_{\mathrm{LFM}}, n=8, \alpha_{1}=-80 \mathrm{~dB}, \mathrm{BT}=100$

FIGURE 5: The frequency modulation law for a signal having a Taylor PSD function.

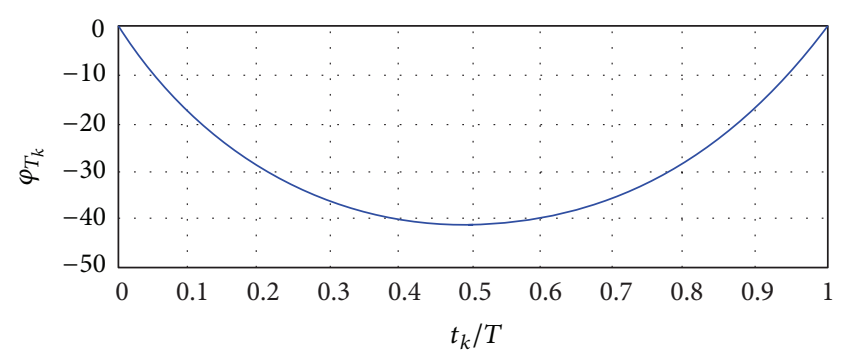

$\Delta F_{\mathrm{LFM}}=1 \mathrm{MHz}, \Delta F_{T}=1.86 \cdot \Delta F_{\mathrm{LFM}}, n=8, \alpha_{1}=-80 \mathrm{~dB}, \mathrm{BT}=100$

FIGURE 6: The phase modulation law for a signal having a Taylor PSD function.

In addition, the real shape assigned to the normalized envelope of the compression filter response obtained by numerical calculus and having as starting point (14) is illustrated in Figure 7.

Compared with previously mentioned PSD functions (e.g., $\cos ^{n}$ and $\cos ^{2}$ on pedestal spectra, truncated Gaussian), the Taylor spectrum window is one more efficient as sidelobe reduction technique. However, in order to conserve the range resolution, a significant widening of the frequency range is necessary (approximately, by 1.86 times).

4.2. Synthesis and Analysis of NLFM Signal Using a PSD Function by Blackman-Harris Type. The PSD function can be written as follows:

$$
\begin{array}{r}
\left|S_{\mathrm{BH}}(j f)\right|^{2}=S_{\mathrm{BH}} \cdot\left[a_{0}+\sum_{k=1}^{3} a_{k} \cdot \cos \left(2 \pi k \cdot \frac{f}{\Delta F_{\mathrm{BH}}}\right)\right], \\
f \in\left[-\frac{\Delta F_{\mathrm{BH}}}{2},+\frac{\Delta F_{\mathrm{BH}}}{2}\right],
\end{array}
$$

where the involved coefficients have the following values: $a_{0}=$ $0.35875, a_{1}=0.48829, a_{2}=0.14128$, and $a_{3}=0.01168$.

The frequency deviation $\Delta F_{\mathrm{BH}}$ will have such value that the range resolution is not worsened compared with the case of LFM radio pulse with frequency deviation $\Delta F_{\mathrm{LFM}}$; namely, $\Delta F_{\mathrm{BH}}=1.12 \cdot \Delta F_{\mathrm{LFM}}$ (Figure 8).
According to (10), the idealized shape assigned to the normalized envelope of the signal autocorrelation function $\rho_{\mathrm{id}_{\mathrm{BH}}}(\cdot)$ will be given by the following equation (Figure 9 ):

$$
\begin{aligned}
& \rho_{\mathrm{BH}}(t)=\operatorname{sinc}\left(\pi \cdot t \cdot \Delta F_{\mathrm{BH}}\right) \\
&+0.5 \cdot \sum_{k=1}^{3} \frac{a_{k}}{a_{0}} \cdot\left\{\operatorname{sinc}\left[\pi \cdot\left(t \cdot \Delta F_{\mathrm{BH}}+k\right)\right]\right. \\
&\left.\quad+\operatorname{sinc}\left[\pi \cdot\left(t \cdot \Delta F_{\mathrm{BH}}-k\right)\right]\right\} .
\end{aligned}
$$

Next, the time of group delay of the signal can be calculated using the following equation:

$$
\begin{aligned}
& T_{g_{\mathrm{BH}}}(f)= \int_{-\infty}^{f}\left|S_{\mathrm{BH}}(j f)\right|^{2} \mathrm{~d} f \\
&=T \cdot\left\{\frac{f}{\Delta F_{\mathrm{BH}}}+\frac{1}{2 \pi}\right. \\
&\left.\cdot \sum_{k=1}^{3}\left[\frac{a_{k}}{k} \cdot \sin \left(2 \pi k \cdot \frac{f}{\Delta F_{\mathrm{BH}}}\right)\right]+0.5\right\} .
\end{aligned}
$$

It can be easily observed that $T_{g_{\mathrm{BH}}}\left(-\Delta F_{\mathrm{BH}} / 2\right)=0$ and $T_{g_{\mathrm{BH}}}\left(+\Delta F_{\mathrm{BH}} / 2\right)=T$, respectively (Figure 10 ).

According to (12), the signal phase-frequency dependency can be written as follows:

$$
\begin{aligned}
\Phi_{s_{\mathrm{BH}}}(f)=-2 \pi \cdot \int_{-\Delta F_{\mathrm{BH}} / 2}^{f} T_{g}(\xi) \mathrm{d} \xi \\
=2 \pi \cdot \Delta F_{\mathrm{BH}} \cdot\left[\frac{1}{(2 \pi)^{2}}\right. \\
\cdot \sum_{k=1}^{3} \frac{a_{k}}{a_{0} \cdot k^{2}} \cdot \cos \left(2 \pi k \cdot \frac{f}{\Delta F_{\mathrm{BH}}}\right) \\
-0.5 \cdot\left(\frac{f}{\Delta F_{\mathrm{BH}}}\right)^{2}-0.5 \cdot \frac{f}{\Delta F_{\mathrm{BH}}} \\
\left.\quad-\frac{1}{(2 \pi)^{2}} \cdot \sum_{k=1}^{3} \frac{a_{k}}{a_{0} \cdot k^{2}} \cdot(-1)^{k}\right] .
\end{aligned}
$$

The frequency modulation law obtained by solving through numerical methods of the equations $T_{g_{\mathrm{BH}}}(f)=t$ is depicted in Figure 11.

The phase modulation law obtained by numerical integration of the frequency modulation law can be calculated according to following equations:

$$
\begin{aligned}
\varphi_{\mathrm{BH}}(t) & =2 \pi \cdot \int_{0}^{t} f_{\mathrm{BH}}(t) \mathrm{d} t \\
& \Longleftrightarrow \varphi_{T_{k}}=2 \pi \cdot \frac{T}{N} \cdot \sum_{i=0}^{k} f_{\mathrm{BH}_{i}}, \quad k=0,1, \ldots, N,
\end{aligned}
$$

and is also depicted in Figure 12. 


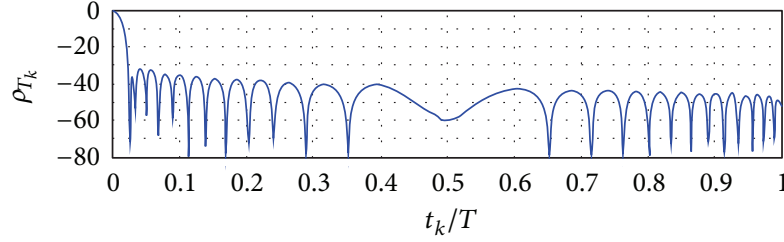

$\Delta F_{\mathrm{LFM}}=1 \mathrm{MHz}, \Delta F_{T}=1.86 \cdot \Delta F_{\mathrm{LFM}}, n=8, \alpha_{1}=-80 \mathrm{~dB}, \mathrm{BT}=100$

(a)

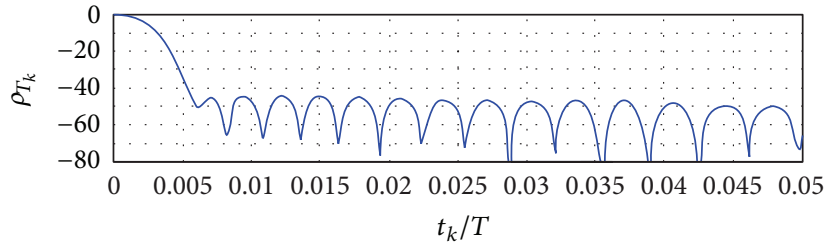

$\Delta F_{\mathrm{LFM}}=5 \mathrm{MHz}, \Delta F_{T}=1.86 \cdot \Delta F_{\mathrm{LFM}}, n=8, \alpha_{1}=-80 \mathrm{~dB}, \mathrm{BT}=500$

(b)

FIGURE 7: The real shape assigned to the normalized envelope of the compression filter response for a signal having a Taylor PSD function.

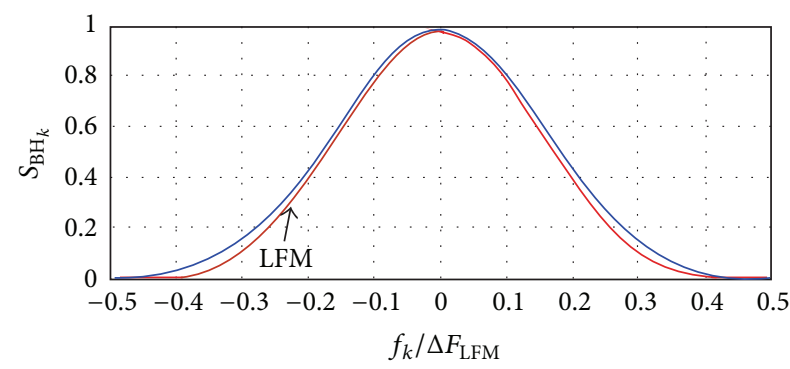

FIGURE 8: Blackman-Harris PSD function.

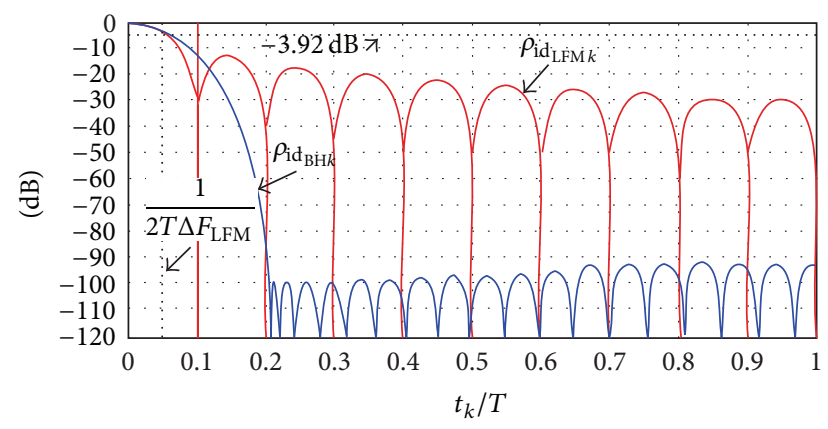

$$
\Delta F_{\mathrm{LFM}}=1 \mathrm{MHz}, \Delta F_{\mathrm{BH}}=1.12 \cdot \Delta F_{\mathrm{LFM}}, \mathrm{BT}=100
$$

FIGURE 9: The normalized envelope of the signal autocorrelation function using the correction of the frequency deviation.

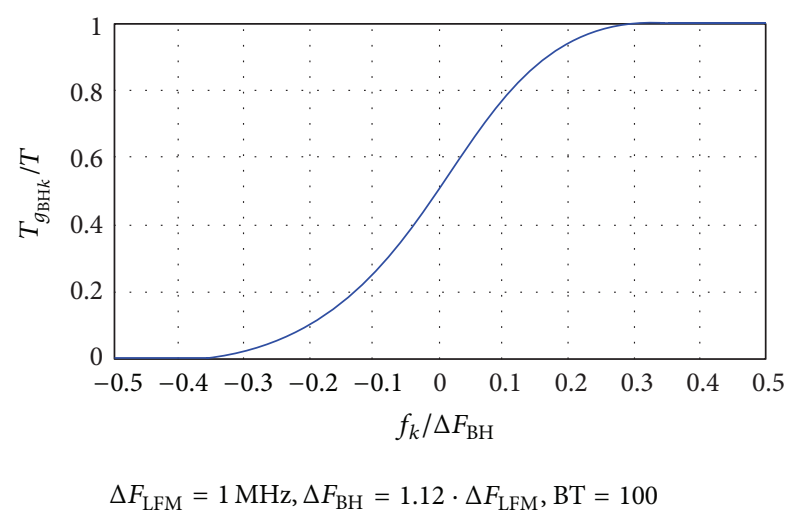

FIGURE 10: Dependency between time of group delay and frequency for a signal having a Blackman-Harris PSD function.

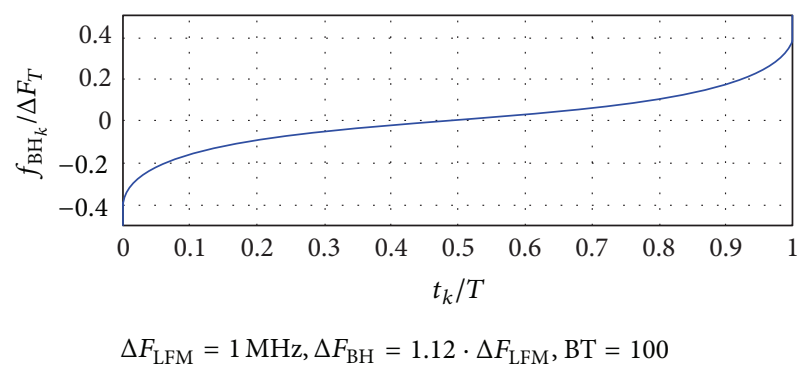

Figure 11: The frequency modulation law for a signal having a Blackman-Harris PSD function.

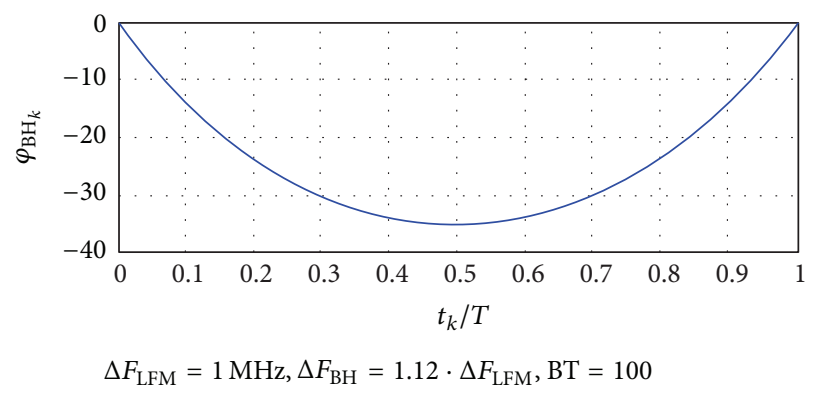

FIGURE 12: The phase modulation law for a signal having a Blackman-Harris PSD function.

In addition, the real shape assigned to the normalized envelope of the compression filter response obtained by numerical calculus and having as starting point (14) is illustrated in Figure 13.

Despite that obtained results are similar with the ones from the case of Taylor window, the spectrum based on Blackman-Harris distribution requires an insignificant widening of the frequency range (approximately, by 1.12 times).

Finally, it is interesting to present a synthetic analysis of the NLFM radio pulse based on ambiguity function tool use. Also, because the experimental results achieving in the two previously analyzed cases are quite similar, only the case of Blackman-Harris spectrum window will be next discussed. Accordingly, based on the discrete values indicated in Figure 12, and computing by a specific numerical method the integral given by (15), a quarter from the (normalized) 


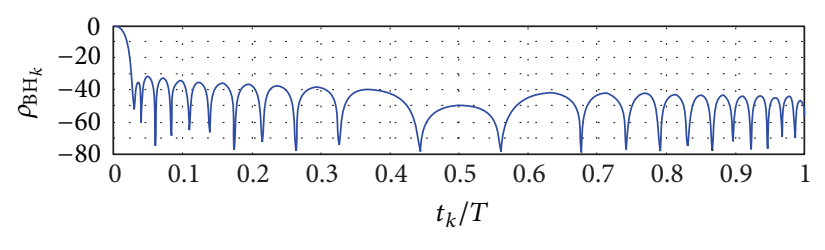

$$
\Delta F_{\mathrm{LFM}}=1 \mathrm{MHz}, \Delta F_{\mathrm{BH}}=1.12 \cdot \Delta F_{\mathrm{LFM}}, \mathrm{BT}=100
$$

(a)

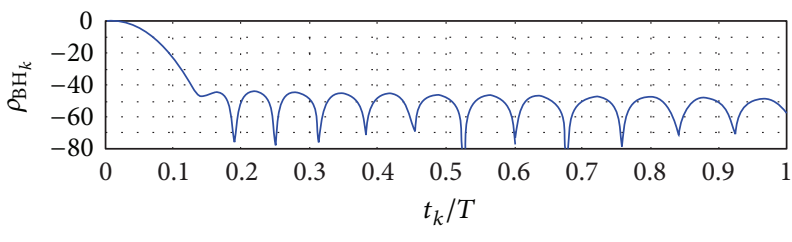

$\Delta F_{\mathrm{LFM}}=5 \mathrm{MHz}, \Delta F_{\mathrm{BH}}=1.12 \cdot \Delta F_{\mathrm{LFM}}, \mathrm{BT}=500$

(b)

FIGURE 13: The real shape assigned to the normalized envelope of the compression filter response for a signal having a Blackman-Harris PSD function.

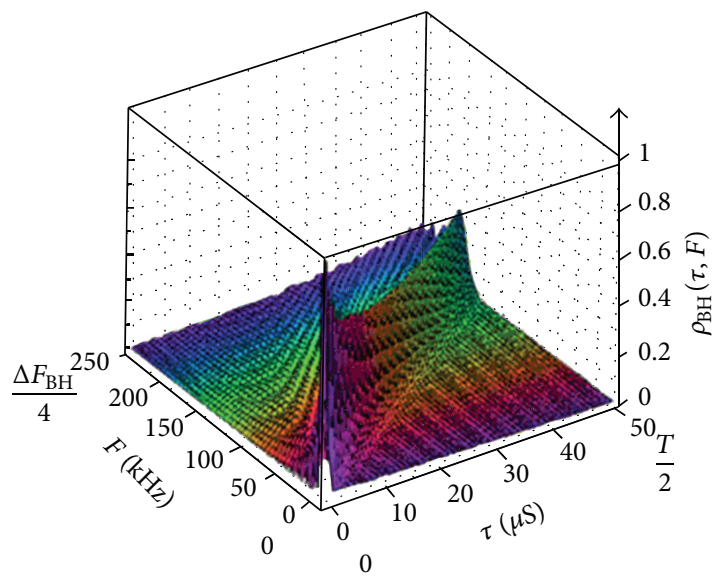

(a) In absence of (for small) Doppler deviation

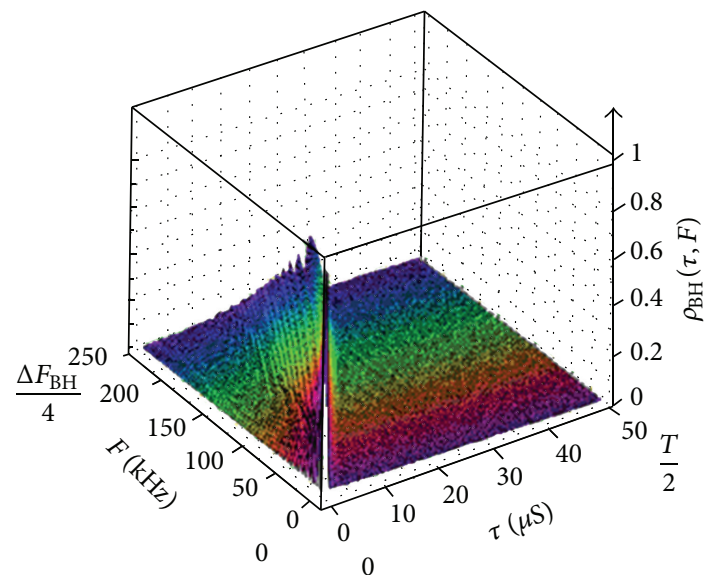

(b) In presence of (significant) Doppler deviation

FIGURE 14: The ambiguity body for a signal having a BlackmanHarris PSD function.

ambiguity body for a signal having a PSD function by Blackman-Harris type is illustrated in Figure 14.

As can be observed in Figure 14 (left side), in absence or for small Doppler deviation, the shape knife blade (which is specific to LFM radio pulse) is preserved but is curved to the axis of Doppler frequency shift $F$ and has a small peak around its origin, respectively. In addition, in presence of Doppler deviation (right side) unfortunately, it can be observed that the achieved response is one deformed, and the level assigned to the sidelobes increases concomitantly with the increasing of the Doppler frequency shift, respectively. Consequently, in order to avoid this disadvantage, some proper technical measures (e.g., in case of a SAR system, it is necessary to compensate the platform shift influence on the phase of echo signal, or to assure the condition $\Delta F \gg F_{\max }$ by a proper projection technique) must be taken $[4,16]$.

\section{Conclusion}

Having as starting point the remark that the level assigned to the sidelobes is determined by the shape of signal spectral density function, and based on stationary phase method, a powerful NLFM synthesis algorithm (i.e., which assures a sidelobe suppression of the autocorrelation function less than $-40 \mathrm{~dB}$, preserves the (LFM) range resolution, and offers null losses related to the optimal filtering/weighting) was theoretically and experimentally described.

Generally, by testing of two promising types of signal PSD functions (i.e., Taylor and Blackman-Harris weighting windows), the first conclusion was that the second window is the most efficient as the sidelobe reduction and widening of the frequency range requirement, all in order to preserve the range resolution similar with the one obtained in case of LFM radio pulse. In addition, the accuracy of the proposed synthesis method increases concomitantly with the signal base (e.g., very good results are achieved for products BT more than 100).

According to modern radar theory, because of the most recent advances in the field of signal processing hardware devices (e.g., FPGA technology, high-speed DACs, etc.), the physical generation of such scanning NLFM radio pulses can become an acceptable task. For example, the newest digital synthesizers can assure synthesis with reduced and controllable clippings of any signal waveform by complex envelope components of the signal.

In summary, the synthesis of NLFM laws using the previously described algorithm has been demonstrated to be a powerful processing technique as sidelobe suppression. More importantly, based on the recent progress reached in the field of signal hardware processing devices, its implementation is feasible and assures a sidelobe reduction level greater than one achieved in case of other consecrated processing (synthesis) methods of radar NLFM signals. 


\section{References}

[1] L. Levanon, E. Mozeson, and E., Radar Signals, John Wiley \& Sons, Hoboken, NJ, USA, 2004.

[2] M. A. Richards, Fundamentals of Radar Signal Processing, McGraw-Hill, New York, NY, USA, 2005.

[3] L. Anton, Signal Processing in High Resolution Radars, MTA Press, Bucharest, Romania, 2008.

[4] G. Iubu, Optimal Processing of Radar Signals, MTA Press, Bucharest, Romania, 1998.

[5] A. W. Doerry, "Generating nonlinear FM chirp waveforms for radar," Tech. Rep., Sandia National Laboratories, 2006.

[6] L. R. Varshney and D. Thomas, "Sidelobe reduction for matched filter range processing," in Proceedings of the IEEE Radar Conference, pp. 446-451, May 2003.

[7] I. Gladkova, "Design of frequency modulated waveforms via the Zak transform," IEEE Transactions on Aerospace and Electronic Systems, vol. 40, no. 1, pp. 355-359, 2004.

[8] C. Leśnik and A. Kawalec, "Modification of a weighting function for NLFM radar signal designing," Acta Physica Polonica A, vol. 114, no. 6, pp. A143-A149, 2008.

[9] B. Zakeri, M. Zahabi, and S. Alighale, "Sidelobes level improvement by using a new scheme used in microwave pulse compression radars," Progress in Electromagnetics Research Letters, vol. 30, pp. 81-90, 2012.

[10] Y. K. Chan, M. Y. Chua, and V. C. Koo, "Sidelobes reduction using simple two and tri-stages non linear frequency modulation (NLFM)," Progress in Electromagnetics Research, vol. 98, pp. 33-52, 2009.

[11] L. Jackson, S. Kay, and N. Vankayalapati, "Iterative method for nonlinear FM synthesis of radar signals," IEEE Transactions on Aerospace and Electronic Systems, vol. 46, no. 2, pp. 910-917, 2010.

[12] I. C. Vizitiu, L. Anton, F. Popescu, and G. Iubu, "Sidelobes reduction using frequency predistortioning techniques on LFM signals," in Proceedings of the IEEE 10th International Symposium on Electronics and Telecommunications (ISETC '12), pp. 381-384, 2012.

[13] F. Cook and M. Bernfeld, Radar Signals, Artech-House, Boston, Mass, USA, 1993.

[14] C. Lesnik, A. Kawalec, and M. Szugajew, The Synthesis of A Radar Signal having Nonlinear Frequency Modulation Function, WIT Press, Southampton, UK, 2011.

[15] S. Boukeffa, Y. Jiang, and T. Jiang, "Sidelobe reduction with nonlinear frequency modulated waveforms," in Proceedings of the IEEE 7th International Colloquium on Signal Processing and Its Applications (CSPA '11), pp. 399-403, March 2011.

[16] I. C. Vizitiu, L. Anton, F. Popescu, and G. Iubu, "The synthesis of some NLFM laws using the stationary phase principle," in Proceedings of the IEEE 10th International Symposium on Electronics and Telecommunications (ISETC '12), pp. 59-62, 2012.

[17] P. Yichun, P. Shirui, Y. Kefeng, and D. Wenfeng, "Optimization design of NLFM signal and its pulse compression simulation," in Proceedings of IEEE Radar Conference, pp. 383-386, 2005.

[18] F. Gran and J. A. Jensen, "Designing NLFM signals for medical ultrasound imaging," in Proceedings of IEEE Ultrasonic Symposium, pp. 1714-1717, 2006.

[19] Y. P. Grishin and A. Zankiewicz, "Neural network sidelobe suppression filter for a pulse-compression radar with powersof-two weights," in Proceedings of the IEEE 10th Mediterranean
Electrotechnical Conference (MALECON '00), pp. 713-716, May 2000.

[20] F. B. Duh, C. F. Juang, and C. T. Lin, "A neural fuzzy network approach to radar pulse compression," IEEE Geoscience and Remote Sensing Letters, vol. 1, no. 1, pp. 15-20, 2004.

[21] P. Wang, H. Meng, and X. Wang, "Suppressing autocorrelation sidelobes of LFM pulse trains with genetic algorithm," Tsinghua Science and Technology, vol. 13, no. 6, pp. 800-806, 2008. 

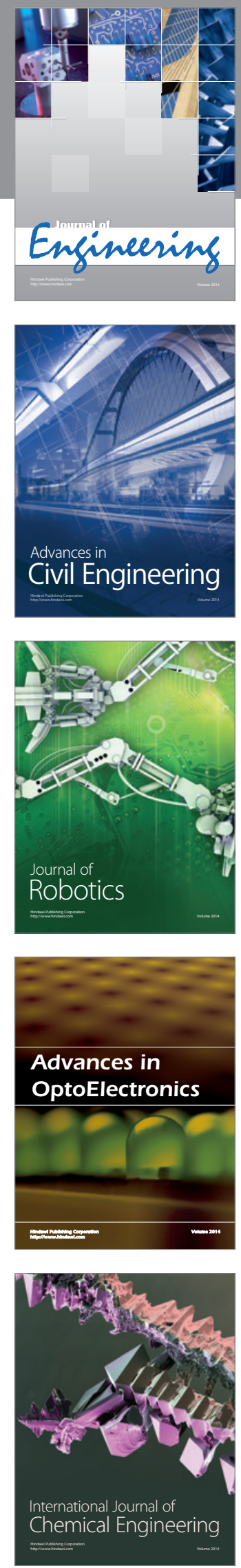

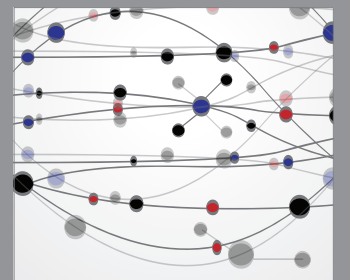

The Scientific World Journal
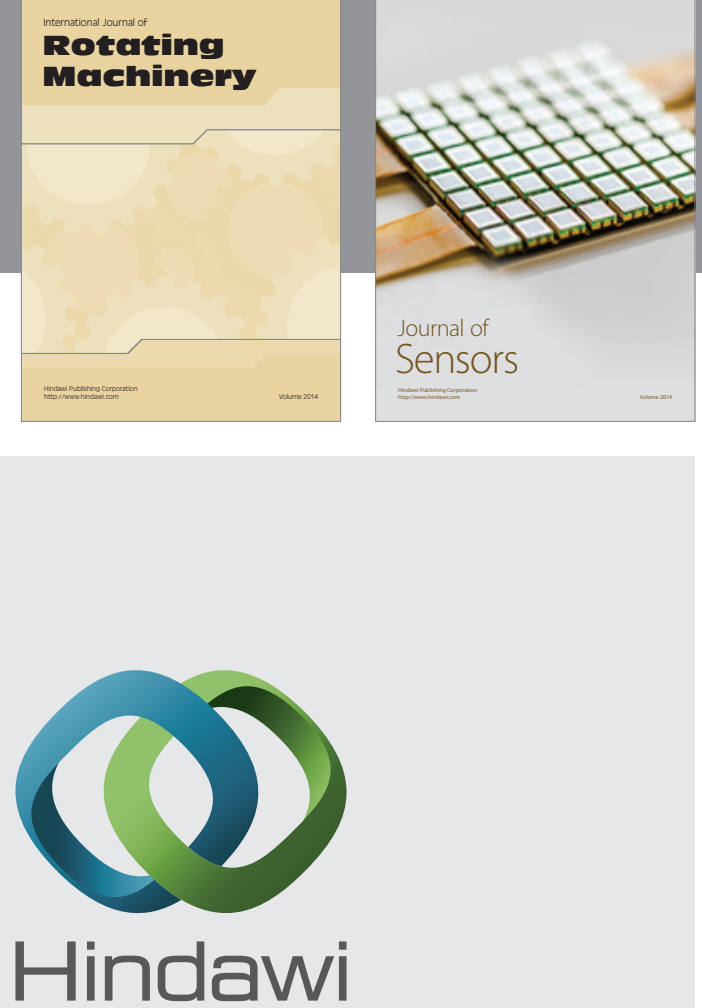

Submit your manuscripts at http://www.hindawi.com
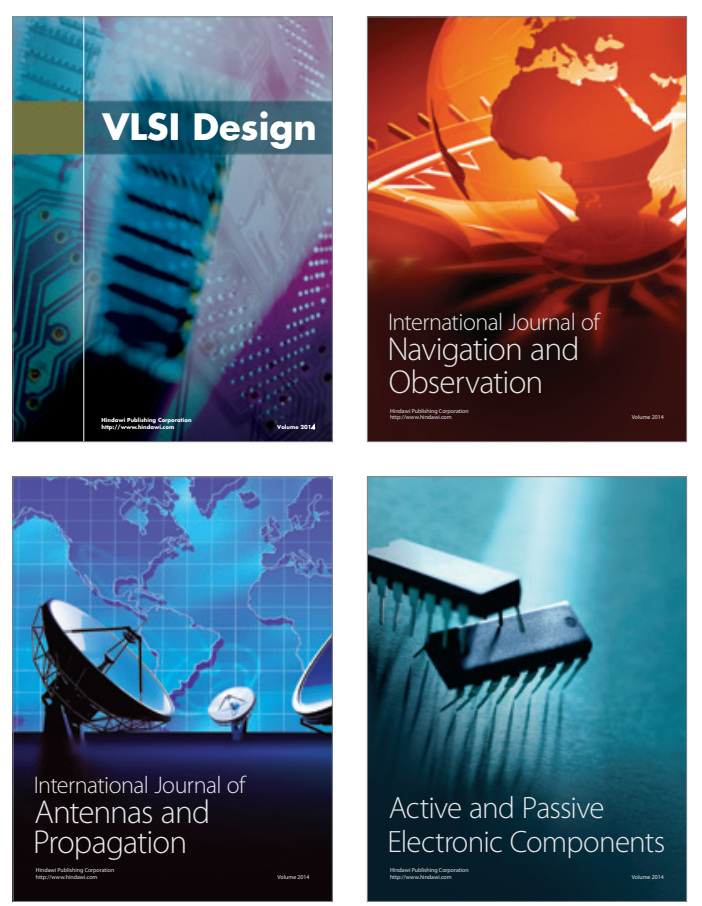
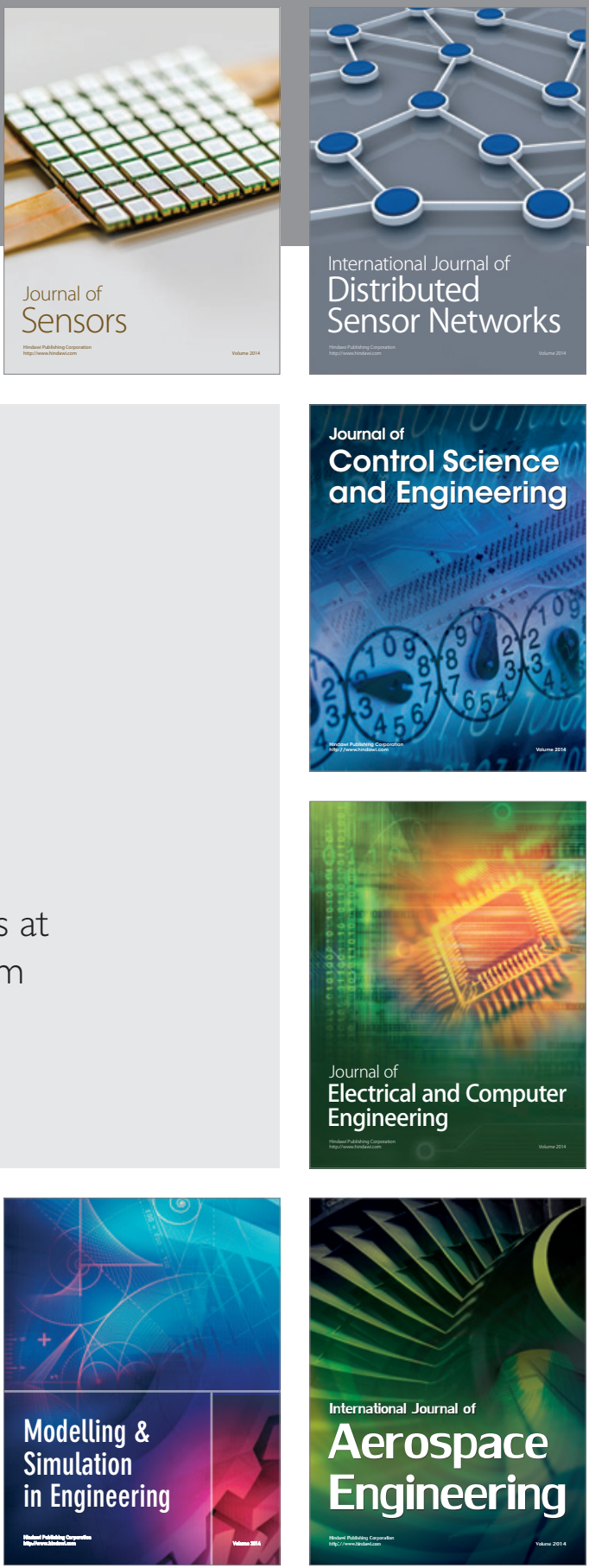

Journal of

Control Science

and Engineering
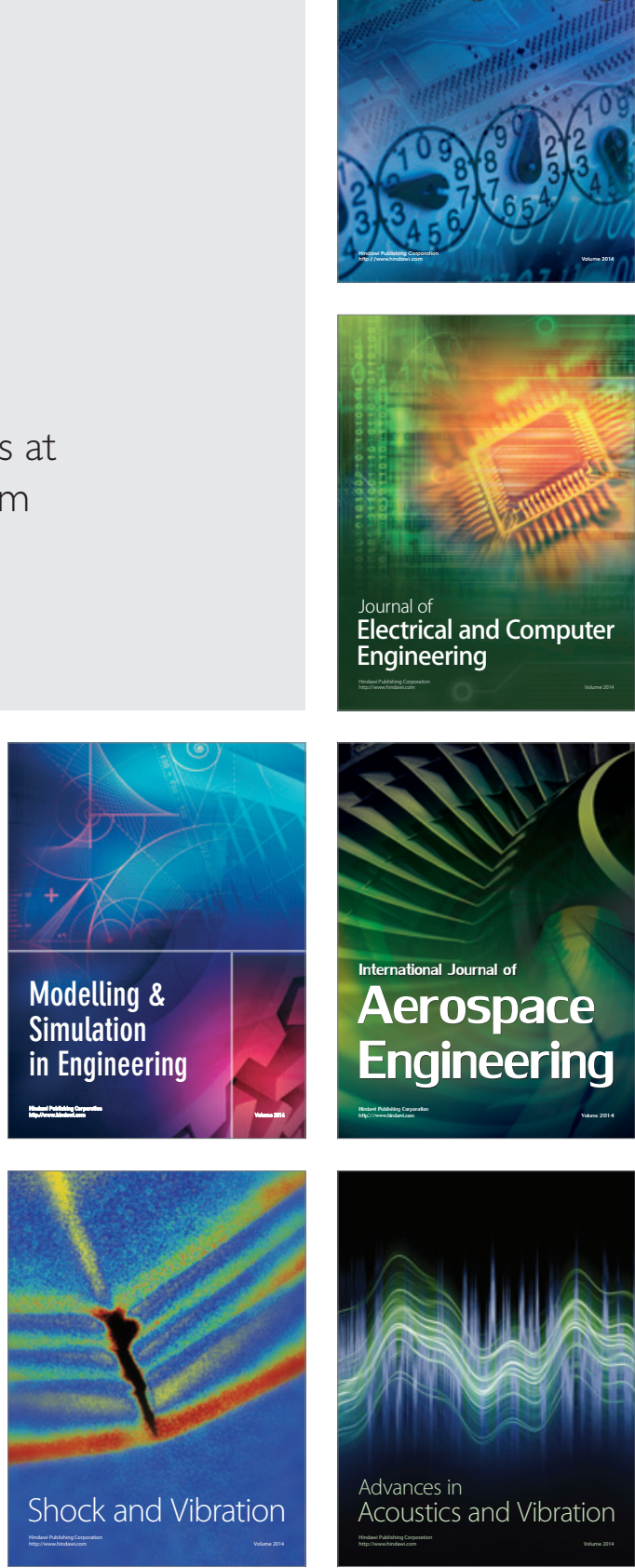